\title{
L'épreuve du chômage: une rupture cumulative des liens sociaux?
}

Serge Paugam

\section{(2) OpenEdition}

1 Journals

Édition électronique

URL : http://journals.openedition.org/ress/248

DOI : $10.4000 /$ ress.248

ISSN : 1663-4446

Éditeur

Librairie Droz

Édition imprimée

Date de publication : 1 août 2006

Pagination : 11-27

ISBN : 9-782-600-01108-2

ISSN : 0048-8046

Référence électronique

Serge Paugam, "L'épreuve du chômage: une rupture cumulative des liens sociaux? », Revue européenne des sciences sociales [En ligne], XLIV-135 | 2006, mis en ligne le 13 octobre 2009, consulté le 10 décembre 2020. URL : http://journals.openedition.org/ress/248 ; DOI : https://doi.org/10.4000/ ress. 248

(c) Librairie Droz 
Serge PAUGAM*

\section{L'ÉPREUVE DU CHÔMAGE: UNE RUPTURE CUMULATIVE DES LIENS SOCIAUX?}

Parce qu'il est souvent considéré comme une des causes majeures de la dégradation du lien social, le chômage mobilise les chercheurs en sciences sociales. Il a été souvent étudié comme un processus de cumul progressif de handicaps. Les recherches ont mis l'accent sur la dégradation du niveau de vie, mais aussi sur l'affaiblissement de la vie sociale et la marginalisation vis-à-vis des autres travailleurs. Les premières grandes enquêtes sociologiques sur ce sujet, notamment celle de Paul Lazarsfeld, Marie Jahoda et de Hans Zeisel à Marienthal en Autriche, datent des années $1930^{1}$ au cours de la période bien connue de crise économique où le chômage avait déjà atteint des sommets. Cette question a fait l'objet de beaucoup moins de recherches pendant la période de prospérité économique d'après guerre tant le chômage était alors un phénomène résiduel. Ce n'est qu'à la fin des années soixante-dix, après deux chocs pétroliers, que les recherches sur le chômage reprirent aussi bien en économie et en sociologie que dans le domaine de la politique sociale.

L'ouvrage de Dominique Schnapper, L'épreuve du chômage, a marqué en France le début d'une nouvelle phase pour ce type de recherches ${ }^{2}$. Il constitue encore aujourd'hui une référence au moins autant pour son contenu - même si la conjoncture économique n'est plus la même - que pour l'intérêt méthodologique qu'il représente. Il permet, en effet, aux étudiants de sociologie de se former à l'enquête par entretiens semi-directifs et à apprendre à élaborer une typologie des expériences vécues ${ }^{3}$. C'est la raison pour laquelle les enseignants continuent, vingt-cinq ans après sa première parution, à en recommander la lecture.

L'enquête à l'origine de ce livre repose sur cent entretiens libres. Il ne s'agit pas comme dans Les chômeurs de Marienthal d'une monographie, mais d'une

* Directeur d'études à l'Ecole des hautes études en sciences sociales, directeur de recherche au CNRS, responsable de l'Equipe de Recherche sur les Inégalités Sociales (ERIS - Centre Maurice Halbwachs); Serge.Paugam@ehess.fr

1 P. Lazarsfeld, M. Jahoda, H. Zeisel, (1933), Marienthal: The Sociology of an Unemployed Community, London, Tavistock (traduction en francais: Les chômeurs de Marienthal, Paris, Editions de Minuit, 1981). Il faut citer aussi celle de E. W. Bakke (1940), The Unemployed Worker : A Study of the Task of Making a Living without a Job, New Haven, Yale University Press.

2 D. Schnapper, L'épreuve du chômage, Paris, Gallimard, 1981 (nouvelle édition Folio, 1994).

3 Dominique Schnapper a expliqué cette méthode en revenant notamment sur L'épreuve du chômage dans son livre La compréhension sociologique, Paris, PUF, 1999, $2^{\mathrm{e}}$ édition, coll. "QuadrigeManuels », 2005. 
enquête qualitative dans laquelle le choix des personnes interrogées a été effectué de façon à avoir un échantillon diversifié des chômeurs, selon le sexe, l'âge, la catégorie socioprofessionnelle et la ville ou la région de résidence. L'objet d'études n'est pas en premier lieu de décrire ou d'inventorier l'ensemble des dimensions de la vie des chômeurs - même si l'enquête permet d'en étudier plusieurs -, mais plus précisément de comprendre le sens que les individus au chômage donnent à leurs expériences vécues.

Dominique Schnapper souligne que ce type de méthode présente l'avantage de dégager les réponses de l'image stéréotypée du «bon chômeur», à laquelle les chômeurs risquent toujours de se référer lorsqu'on les interroge de façon sommaire dans des sondages d'opinion. «Elle permet, grâce à la confiance de l'interviewé, d'obtenir sinon l'expérience vécue, par définition inaccessible, du moins une expression qui en est proche. Il est plus facile de faire admettre, au cours d'un long entretien amical, les avantages du chômage ou simplement de faire exprimer et analyser les sentiments de ceux qui, par leur style personnel ou leur appartenance sociale, sont peu disposés à le faire.» ${ }^{4}$ Mais l'avantage indéniable de cette méthode n'élimine pas pour autant ses limites. «Ce matériel autorise une analyse purement qualitative, permettant de préciser les divers éléments de l'expérience vécue du chômage, d'expliquer les facteurs qui influencent cette expérience, sans qu'on puisse évaluer le poids relatif de ces facteurs. Il permet, certes, d'élaborer des types, mais indépendamment de leur représentation statistique dans la population globale des chômeurs. Il n'autorise pas à évaluer la part des chômeurs inscrits à l'ANPE qui vivent tel ou tel forme du chômage. [...] Il est même impossible de faire le lien entre les analyses que nous présentons et les enquêtes statistiques qui existent par ailleurs, sinon par l'intermédiaire de la catégorie socioprofessionnelle.» ${ }^{5}$

Je voudrais ici, dans le cadre de ces mélanges en hommage à Dominique Schnapper, partir de ce livre pour aborder une question sociologique importante: celle de la rupture cumulative des liens sociaux. Le chômage, qui correspond déjà en soi à une rupture du lien qui rattache l'individu au monde du travail, est-il susceptible d'en entraîner d'autres, en particulier une rupture du lien familial ou une rupture des relations affinitaires avec les amis, les proches, les voisins ou encore une rupture avec les modes de participation à la vie institutionnelle d'un pays?

\section{QUE FAUT-IL ENTENDRE PAR RUPTURE DES LIENS SOCIAUX?}

Le propre de la socialisation est de permettre à chaque individu de tisser, à partir de la trame que lui offrent les institutions sociales, les fils de ses appartenances multiples qui lui garantissent le confort de la protection et l'assurance de la reconnaissance sociale. Mais ce tissage n'est pas identique d'un individu à un autre. Dans certains cas, les fils sont tous faibles et le maillage social très fragile. Dans d'autres cas, certains fils sont plus solides que d'autres, mais le tissu n'est pas

\footnotetext{
4 Cf. L'épreuve du chômage, op. cit, p. 59.

5 Ibid., p. 60.
} 
pour autant à l'abri d'accrocs et, petit à petit, de trous. En réalité, dans une étoffe où les fils sont entrecroisés, le risque est toujours que la rupture de l'un d'entre eux entraîne un effilochage et, progressivement, par la pression exercée à l'endroit précis de la faiblesse, la rupture des autres. C'est en ce sens que le chômage constitue un risque de désocialisation.

Dans L'épreuve du chômage, Dominique Schnapper distingue trois types d'expériences vécues: le chômage total, le chômage inversé, le chômage différé. Le chômage total se caractérise par l'ennui, la désocialisation, l'humiliation. Il constitue l'expérience de la grande majorité des travailleurs manuels, de certains employés et dans une moindre mesure de cadres d'origine modeste, c'est-à-dire de tous ceux pour lesquels le travail représente le mode privilégié de l'expression de soi au sens où il apporte non seulement une activité et une rémunération, mais une raison d'être, un sentiment d'utilité et une reconnaissance sociale. Le chômage inversé est vécu de façon très différente, essentiellement par une population jeune, d'origine sociale moyenne ou même supérieure. Cette expérience peut correspondre à des vacances prolongées pour des personnes qui n'ont pas connu la vie professionnelle ou qui retrouvent, après une brève expérience de travail, le charme de l'oisiveté ou le plaisir d'avoir du temps pour soi ou pour ses loisirs. Certains profitent de cette expérience pour se consacrer à leur passion et dans certains cas à leur vie d'artiste. Enfin, le chômage différé, celui qui correspond à la catégorie des cadres, se caractérise par la recherche active d'un emploi ou l'adoption d'activités de substitution. La plupart des cadres qui font cette expérience tiennent à maintenir les normes de l'univers professionnel et s'accrochent au statut de «cadre en chômage», ce qui permet de différer au-delà d'un an l'apparition des traits propres au chômage total.

De ces trois types d'expériences vécues, seul le premier, le chômage total semble vérifier la tendance à la rupture cumulative des liens sociaux. Certes, les entretiens recueillis auprès des chômeurs qui font cette expérience ne permettent pas de valider entièrement la thèse d'une spirale descendante conduisant inexorablement à l'isolement social - seule une enquête longitudinale permet vraiment de le démontrer -, mais les extraits cités expriment une grande souffrance et un processus très net de désocialisation. Des expressions comme: «je ne sors presque plus maintenant», «je ne vois presque plus personne», «on s'est complètement perdu, on est perdu », «non, je ne cherche plus à voir les autres » sont très courantes dans la bouche de ces hommes et de ces femmes en proie au désespoir. Pour Dominique Schnapper, c'est cette expérience du chômage total, en tant qu'expérience traumatisante pour la grande majorité de la population, qui constitue le type idéal de l'épreuve du chômage, les deux autres pouvant être considérées comme des déviations.

Le chômage constitue donc déjà une rupture d'un lien. Quelle est la spécificité de ce dernier par rapport aux autres liens sociaux? Il est possible de distinguer quatre grands types de liens sociaux: le lien de filiation, le lien de participation élective, le lien de participation organique et le lien de citoyenneté. Parler de lien de filiation, c'est reconnaître que chaque individu naît dans une famille et rencontre en principe à sa naissance à la fois son père et sa mère ainsi qu'une famille élargie à laquelle il appartient sans qu'il l'ait choisie, mais c'est aussi insister sur la fonction socialisatrice de la famille. Le lien de filiation contribue à l'équilibre affectif de l'individu dès sa naissance puisqu'il lui assure à la fois stabilité et 
protection. Les psychologues ont démontré que chaque enfant éprouvait des pulsions d'attachement qui nécessitaient d'être satisfaites. La solidarité entre les parents et les enfants constitue une forme d'expression de ce lien. Cette solidarité est toutefois fragile et peut se défaire à l'occasion de l'épreuve du chômage.

Le lien de participation élective relève de la socialisation extra-familiale au cours de laquelle l'individu entre en contact avec d'autres individus qu'il apprend à connaître dans le cadre de groupes divers et d'institutions. Pour participer à la vie sociale en dehors de son cadre familial, il faut qu'il s'intègre en apprenant à respecter des normes et des règles qui lui préexistaient. Les lieux de cette socialisation sont nombreux: le voisinage, les bandes, les groupes d'amis, les communautés locales, les institutions religieuses, sportives, culturelles, etc. Au cours de ses apprentissages sociaux, l'individu est à la fois contraint par cette nécessité de s'intégrer, mais il est en même temps en partie autonome dans le sens où il peut construire lui-même son réseau d'appartenances à partir duquel il pourra affirmer sa personnalité sous le regard des autres. On peut considérer la formation du couple comme un lien de participation élective. L'individu s'intègre par cet acte à un autre réseau familial que le sien. Il élargit ainsi son cercle d'appartenance. Autant dans le lien de filiation, l'individu n'a aucune liberté de choix, autant dans le lien de participation élective, il dispose d'un espace d'autonomie qui lui permet de s'allier et de s'opposer. Il n'existe pas, on le sait, d'alliance qui ne soit en même temps distinction, voire opposition. Autrement dit, l'individu au cours de cette socialisation extra-familiale apprend autant à s'allier à d'autres qu'à se distinguer des autres, voire à s'y opposer. L'expérience du chômage constitue une épreuve pour ce lien de participation élective. Le chômage total analysé par Dominique Schnapper en est l'expression.

Le lien de participation organique relève aussi de la socialisation extra-familiale, mais se distingue du précédent en ce qu'il se caractérise par l'apprentissage et l'exercice d'une fonction déterminée dans l'organisation du travail. Selon Durkheim, ce qui fait le lien social dans les sociétés modernes - ce qu'il appelle la solidarité organique -, c'est avant tout la complémentarité des fonctions, laquelle confère à tous les individus, aussi différents soient-ils les uns des autres, une position sociale précise susceptible d'apporter à chacun à la fois la protection élémentaire et le sentiment d'être utile. Ce lien de participation organique se constitue par conséquent dans le cadre de l'école et se prolonge dans le monde du travail. Le chômage est donc en ce sens une rupture au moins partielle de ce lien de participation organique.

Enfin, le lien de citoyenneté repose sur le principe de l'appartenance à une nation. En théorie, la nation reconnaît à ses membres des droits et des devoirs et en fait des citoyens à part entière. Dans les sociétés démocratiques, les citoyens sont égaux en droit, ce qui implique, non pas que les inégalités économiques et sociales disparaissent, mais que des efforts soient accomplis dans la nation pour que tous les citoyens soient traités de façon équivalente et forment ensemble un corps ayant une identité et des valeurs communes ${ }^{6}$. Le lien de citoyenneté est en quelque sorte supérieur aux autres, puisqu'il est censé dépasser et transcender tous

6 Cf. D. Schnapper, La communauté des citoyens. Sur l'idée moderne de nation, Paris, Gallimard, coll. «nrf/essais », 1994. 
les clivages, les oppositions et les rivalités. Dans les sociétés démocratiques, le citoyen dispose aussi de droits économiques et sociaux qui en font autre chose qu'une simple marchandise. Par ce processus de "démarchandisation", le lien de citoyenneté s'est en quelque sorte élargi pour garantir aux individus une plus grande protection face aux aléas de l'existence.

Ces quatre types de liens sont de nature différente, mais ils ont deux fondements communs. Ils apportent tous aux individus à la fois la protection et la reconnaissance nécessaires à leur existence sociale. La protection renvoie à l'ensemble des supports que l'individu peut mobiliser face aux aléas de la vie (ressources familiales, communautaires, professionnelles, sociales...), la reconnaissance renvoie à l'interaction sociale qui stimule l'individu en lui fournissant la preuve de son existence et de sa valorisation par le regard de l'autre ou des autres.

Ces quatre types de liens sont complémentaires et entrecroisés. Ils constituent en quelque sorte, par leur entrecroisement, le tissu social qui enveloppe l'individu. Lorsque ce dernier décline son identité à des personnes qu'il rencontre pour la première fois, il peut faire référence aussi bien à sa nationalité (lien de citoyenneté), à sa profession (lien de participation organique), à ses groupes d'appartenance (lien de participation élective), à ses origines familiales (lien de filiation). Ces quatre types de liens peuvent être relativement différents d'une société à l'autre. Dans chaque société, ils constituent toutefois la trame sociale qui préexiste aux individus et à partir de laquelle ils sont appelés à tisser leurs appartenances au corps social par le processus de socialisation. Si l'intensité de ces liens sociaux varie d'un individu à l'autre en fonction des conditions particulières de sa socialisation, elle dépend aussi de l'importance relative que les sociétés leur accordent. Le rôle que jouent par exemple les solidarités familiales et les attentes collectives à leur égard sont variables d'une société à l'autre. Les formes de sociabilité qui découlent du lien de participation élective ou du lien de participation organique dépendent en grande partie du genre de vie et sont par conséquent plurielles. L'importance accordée au principe de citoyenneté comme fondement de la protection sociale n'est pas équivalent dans tous les pays.

\section{CHÔMAGE ET ISOLEMENT FAMILIAL}

Le chômage, en tant que rupture au moins partielle du lien de participation organique, en entraîne-t-il d'autres? Des travaux récents sur l'expérience du chômage en Europe ont permis d'approfondir cette question et d'étudier les variations entre pays. Dans le cas du programme de recherche que j'ai coordonné avec Duncan Gallie ${ }^{7}$, nous avons eu recours au European Community Household Panel (ECHP) réalisé de 1994 à 2001 (soit huit vagues) auprès d'un échantillon de ménages représentatif de chacun des 12 pays de 1'Union Européenne ${ }^{8}$. Cette

7 Cf. D. Gallie et S. Paugam (dir.), Welfare Regimes and the Experience of Unemployment in Europe, Oxford, Oxford University Press, 2000.

8 Lors de la première vague, l'échantillon total comportait 60500 ménages, c'est-à-dire approximativement 130000 adultes âgés de 16 ans et plus. 
source n'a pas été élaborée pour étudier spécifiquement le chômage, mais elle comporte de nombreuses questions sur l'expérience professionnelle, le rapport au travail, les conditions de vie du ménage et quelques éléments sur les solidarités familiales, la vie sociale (relations avec les amis, les voisins, la participation à la vie associative $)^{9}$.

Prenons tout d'abord la probabilité de vivre seul. Il ne s'agit pas en soi d'un indicateur de fragilité des réseaux sociaux. On peut y voir, en effet, un indice d'autonomie choisie des individus vis-à-vis de la famille et de leur entourage. Cette autonomie n'empêche pas des liens étroits avec les parents, les amis et n'est pas contradictoire avec une sociabilité dense et diversifiée. En revanche, si les personnes qui vivent seules ont également une très faible participation à la vie sociale, le risque d'isolement, voire de repli sur soi est plus grand et on peut craindre alors un processus de disqualification sociale.

En réalité, la probabilité de vivre seul est très variable en Europe d'un pays à l'autre. La proportion de personnes dans cette situation passe en effet de $4 \%$ en Espagne à 22\% au Danemark ${ }^{10}$. Les personnes en dessous du seuil de pauvreté ${ }^{11}$ vivent beaucoup plus fréquemment seules dans les pays du Nord de l'Europe que dans les pays du Sud. Parmi les chômeurs en situation de pauvreté, on trouve près de $41 \%$ de personnes vivant seules au Danemark, 37\% en Allemagne contre 2,2\% en Espagne et 1,4\% en Italie. Notons aussi que plus la situation par rapport à l'emploi est dégradée ${ }^{12}$, plus la probabilité de vivre seul est élevée dans plusieurs pays: $30,2 \%$ des chômeurs de plus d'un an sont dans cette situation au Danemark contre $15.2 \%$ des personnes ayant un emploi stable, soit un écart de 15 points. La proportion de chômeurs de plus d'un an vivant seuls est également élevée aux PaysBas (23,5\%), en Allemagne (17,5\%) et en Grande-Bretagne (15,7\%, cf. tableau 1). L'écart entre les chômeurs de plus d'un an et les personnes en emploi stable est environ de 10 points aux Pays-Bas et de 8 points en Grande-Bretagne. Cela constitue un contraste avec les pays du Sud de l'Europe où la proportion de chômeurs de plus d'un an vivant seuls est très faible: moins de $2 \%$ en Espagne et au Portugal et moins de $4 \%$ en Italie et en Grèce.

${ }^{9}$ Il s'agit cependant le plus souvent de questions objectives. Les opinions, les représentations et les expériences subjectives ne sont pas abordées. En revanche, le caractère européen et longitudinal de cette enquête permet de faire des comparaisons entre pays et d'étudier les effets du chômage dans la durée.

${ }^{10}$ Cf. J. Middlemans et R. Paserman, Vivre sous le même toit. Modèles familiaux dans l'Union Européenne, Insee Première, 43, 1996, pp. 1-4.

${ }^{11}$ Selon le seuil de $60 \%$ du revenu médian, échelle de l'OCDE modifiée.

${ }^{12}$ Les différentes situations par rapport à l'emploi distinguées dans cette section ont été notamment établies à partir d'une question du Panel européen des ménages portant sur la satisfaction des actifs à l'égard de la stabilité de l'emploi. Ceux qui étaient satisfaits ont été considérés comme ayant un emploi stable. Ceux qui n'étaient pas satisfaits, mais qui avaient leur emploi depuis plus d'un an ont été considérés comme ayant un emploi menacé. Ceux qui n'étaient pas satisfaits et qui avaient leur emploi depuis moins d'un an ont été classés comme ayant un emploi instable. Par ailleurs, les chômeurs ont été distingués selon qu'ils faisaient cette expérience depuis moins d'un an ou depuis plus d'un an. 
Dans les pays du Sud de l'Europe, les chômeurs restent dans leur famille tant qu'ils ne peuvent pas fonder un foyer. Il est possible, dans ce cas, de parler d'un modèle de cohabitation familiale de longue durée.

Tableau 1 : Proportion de personnes vivant seules selon la situation par rapport à l'emploi et le pays (en\%).

\begin{tabular}{|c|c|c|c|c|}
\hline Pays & Emploi stable & $\begin{array}{l}\text { Chômage de } \\
\text { moins d'un an }\end{array}$ & $\begin{array}{l}\text { Chômage de } \\
\text { plus d'un an }\end{array}$ & $\begin{array}{l}\text { Ensemble de la } \\
\text { population active } \\
\text { de } 18 \text { à } 65 \text { ans }\end{array}$ \\
\hline $\begin{array}{l}\mathbf{1}^{\text {er }} \text { groupe } \\
\text { Danemark } \\
\text { Pays-Bas }\end{array}$ & $\begin{array}{l}15,2 \\
13,2\end{array}$ & $\begin{array}{l}29,9 \\
19,3\end{array}$ & $\begin{array}{l}30,2 \\
23,5\end{array}$ & $\begin{array}{l}16,5 \\
15,0\end{array}$ \\
\hline $\begin{array}{l}2^{\mathrm{e}} \text { groupe } \\
\text { Allemagne } \\
\text { France } \\
\text { Belgique }\end{array}$ & $\begin{array}{r}15,1 \\
10,6 \\
9,3 \\
\end{array}$ & $\begin{array}{l}21,1 \\
13,0 \\
10,0\end{array}$ & $\begin{array}{r}17,2 \\
10,3 \\
7,9\end{array}$ & $\begin{array}{r}15,4 \\
10,3 \\
9,4\end{array}$ \\
\hline $\begin{array}{l}\mathbf{3}^{\mathbf{e}} \text { groupe } \\
\text { Roy. Uni } \\
\text { Irlande }\end{array}$ & $\begin{array}{l}7,9 \\
8,2\end{array}$ & $\begin{array}{r}15,0 \\
5,0\end{array}$ & $\begin{array}{r}15,7 \\
7,1\end{array}$ & $\begin{array}{l}9,1 \\
7,6\end{array}$ \\
\hline $\begin{array}{l}\mathbf{4}^{\mathbf{e}} \text { groupe } \\
\text { Italie } \\
\text { Espagne } \\
\text { Grèce } \\
\text { Portugal }\end{array}$ & $\begin{array}{l}6,3 \\
3,7 \\
5,8 \\
2,4\end{array}$ & $\begin{array}{l}2,5 \\
1,7 \\
4,5 \\
1,4\end{array}$ & $\begin{array}{l}2,4 \\
1,5 \\
3,7 \\
1,1\end{array}$ & $\begin{array}{l}5,6 \\
3,0 \\
5,3 \\
2,4\end{array}$ \\
\hline
\end{tabular}

Il existe aussi de saisissants contrastes régionaux au sein de ces pays, notamment en Italie. Le niveau de développement économique a une forte incidence sur la structure du chômage, mais il en a aussi sur les structures familiales. Il suffit de prendre la proportion d'enfants de 18 à 30 ans vivant avec leurs parents comme indicateur du caractère traditionnel ou non du modèle familial pour s'en rendre compte. Cette proportion est, en effet, nettement plus élevée dans le Sud de l'Italie que dans le Centre et le Nord, à la fois parmi l'ensemble de la population en âge de travailler et parmi la population des chômeurs. On peut donc confirmer à partir de l'exemple de l'Italie que l'autonomie vis-à-vis de la famille croît avec le niveau de développement économique. Lorsque les possibilités d'emploi sont restreintes, le risque de pauvreté est plus élevé, il devient alors essentiel de conserver des relations avec les membres de sa famille pour faire face aux difficultés de l'existence. Cette explication qui met l'accent sur le poids des contraintes est cependant insuffisante. Comment expliquer alors que tous les jeunes Européens confrontés au chômage ne résident pas chez leurs parents?

Il faut tenir compte de deux facteurs complémentaires. Premièrement, il existe dans les pays du Sud de l'Europe une tradition de solidarité familiale plus répandue que dans les autres pays. Ces solidarités qui s'imposent notamment aux parents sont renforcées à l'intérieur du ménage par une forte division du travail. 
Le chef de famille est le plus souvent l'homme dont le rôle essentiel est d'assurer l'autonomie financière du ménage en y apportant notamment les ressources de son activité professionnelle, tandis que la femme se consacre à l'organisation de la vie domestique et aux enfants, même après que ces derniers ont atteint l'âge adulte.

L'obligation normative de la cohabitation prolongée concerne aussi bien les parents que les enfants. Ces derniers ne peuvent pas vraiment accéder à une autonomie résidentielle et à une vie de couple tant qu'ils n'ont pas obtenu la garantie d'un emploi ou d'une activité professionnelle stable. Il leur paraît alors normal de résider chez leurs parents et ils participent pleinement à la vie du ménage. Dans son enquête sur les jeunes en France, en Grande-Bretagne, au Danemark et en Espagne, Cécile Van de Velde a constaté que les jeunes Espagnols se distinguaient fortement des autres. La rhétorique que ces derniers mobilisent pour justifier leur maintien tardif au foyer de leurs parents est fondée sur une logique d'appartenance familiale ${ }^{13}$. La cohabitation prolongée est normalisée et non stigmatisée. Ils sont nombreux à exprimer une sensation de bien-être chez leurs parents. Ce maintien au foyer est encouragé par les parents eux-mêmes. Un départ prématuré sans raison apparemment légitime serait assimilé à une «trahison affective». Les jeunes Espagnols entendent, de leur côté, «ne pas blesser les parents», de «ne pas trahir l'honneur familial».

Dans ces conditions, le risque d'isolement social est moindre, en particulier pour les jeunes chômeurs. Les spécialistes de la famille ont souvent souligné le rôle important des valeurs familiales et des relations entre parents et enfants dans les pays du Sud de l'Europe et notamment en Italie. On a souvent dit, peut-être trop fréquemment, que l'Italie est avant tout «une collection de familles et non un Etat». Il convient en tout cas de souligner que dans les pays où la famille constitue le fondement privilégié de l'intégration sociale, la pauvreté, ainsi que le chômage, ne se traduisent pas par la faiblesse des liens familiaux.

Un autre facteur d'explication est que les pays du Sud de l'Europe connaissent un plus faible développement du système de protection sociale que les pays du Nord. Dans le domaine de la prise en charge du chômage par exemple, ils se caractérisent par un système que l'on peut qualifier de «sous-régime de protection ${ }^{14}$. Celui-ci n'offre que de très faibles indemnités aux chômeurs, en particulier lorsqu'ils n'ont jamais travaillé, ce qui ne peut qu'accroître leur dépendance à l'égard de leurs familles. Les jeunes chômeurs n'ont donc pas d'autre solution que de résider chez leurs parents en attendant que leur situation professionnelle leur permette d'être autonomes.

Pour apprécier la force des solidarités familiales dans les pays du Sud de l'Europe, on peut prendre en compte également la possibilité des personnes d'être aidées par des membres de leur famille ou de leur entourage ne résidant pas avec elles (cf. tableau 2). Il s'agit ici d'une solidarité qui renvoie non plus à la notion de maisonnée, mais à celle plus large de parentèle. Les données disponibles concernent

${ }^{13}$ Cf. C. Van de Velde, Devenir adulte. Sociologie comparée de la jeunesse en Europe, thèse de doctorat de sociologie, Paris, Institut d'Etudes Politiques de Paris, 2004.

${ }^{14}$ Cf. D. Gallie and S. Paugam (eds), Welfare Regimes and the Experience of Unemployment in Europe, op. cit. 
Tableau 2: Effets de l'instabilité de l'emploi et du chômage sur la probabilité de recevoir une aide financière ou une pension de parents, d'amis ou d'autres personnes vivant en dehors du ménage (régression logistique)

Référence : emploi stable.

\begin{tabular}{|c|c|c|c|c|c|c|c|c|}
\hline \multirow[b]{2}{*}{ Pays } & \multicolumn{2}{|c|}{ Emploi menacé } & \multicolumn{2}{|c|}{ Emploi instable } & \multicolumn{2}{|c|}{ Chômage $<1$} & \multicolumn{2}{|c|}{ Chômage>1 } \\
\hline & B. & Sig. & B. & Sig. & B. & Sig. & B. & Sig \\
\hline $\begin{array}{l}\mathbf{1}^{\text {er }} \text { groupe } \\
\text { Danemark } \\
\text { Pays-Bas }\end{array}$ & $\begin{array}{l}-.03 \\
-.33\end{array}$ & $\begin{array}{l}\text { n.s. } \\
\text { n.s. }\end{array}$ & $\begin{array}{l}-.25 \\
1.23\end{array}$ & $\begin{array}{l}\text { n.s. } \\
* *\end{array}$ & $\begin{array}{l}-.17 \\
-.28\end{array}$ & $\begin{array}{l}\text { n.s. } \\
\text { n.s. }\end{array}$ & $\begin{array}{l}-.04 \\
1.60\end{array}$ & $\begin{array}{l}\text { n.s. } \\
* *\end{array}$ \\
\hline $\begin{array}{l}2^{\mathrm{e}} \text { groupe } \\
\text { Allemagne } \\
\text { France } \\
\text { Belgique }\end{array}$ & $\begin{array}{l}-.06 \\
-.03 \\
-.10\end{array}$ & $\begin{array}{l}\text { n.s. } \\
\text { n.s. } \\
\text { n.s. }\end{array}$ & $\begin{array}{l}-.13 \\
-.50 \\
-.50\end{array}$ & $\begin{array}{l}\text { n.s. } \\
* \\
(*)\end{array}$ & $\begin{array}{l}-.54 \\
-.52 \\
-.02\end{array}$ & $\begin{array}{l}(*) \\
* \\
\text { n.s. }\end{array}$ & $\begin{array}{l}-.35 \\
-.00 \\
-.69\end{array}$ & $\begin{array}{l}(*) \\
\text { n.s. } \\
* *\end{array}$ \\
\hline $\begin{array}{l}\mathbf{3}^{\mathrm{e}} \text { groupe } \\
\text { Roy.Uni } \\
\text { Irlande }\end{array}$ & $\begin{array}{l}-.27 \\
-.02\end{array}$ & $\begin{array}{l}\text { n.s. } \\
\text { n.s. }\end{array}$ & $\begin{array}{l}-.54 \\
-.84\end{array}$ & $\begin{array}{l}* * \\
\text { n.s. }\end{array}$ & $\begin{array}{l}-.10 \\
1.43\end{array}$ & $\begin{array}{l}\text { n.s. } \\
*\end{array}$ & $\begin{array}{l}-.17 \\
-.48\end{array}$ & $\begin{array}{l}\text { n.s. } \\
\text { n.s. }\end{array}$ \\
\hline $\begin{array}{l}\mathbf{4}^{\mathrm{e}} \text { groupe } \\
\text { Italie } \\
\text { Espagne } \\
\text { Grèce } \\
\text { Portugal }\end{array}$ & $\begin{array}{l}-.23 \\
-.28 \\
-.19 \\
-.45\end{array}$ & $\begin{array}{l}(*) \\
\text { n.s. } \\
\text { n.s. } \\
\text { n.s. }\end{array}$ & $\begin{array}{l}-.94 \\
-.64 \\
-.94 \\
-.53\end{array}$ & $\begin{array}{l}* * * \\
* * \\
* * * \\
\text { n.s. }\end{array}$ & $\begin{array}{l}1.09 \\
-.72 \\
-.68 \\
1.38\end{array}$ & $\begin{array}{l}* * * \\
* * \\
* \\
* *\end{array}$ & $\begin{array}{l}1.40 \\
1.20 \\
1.59 \\
1.34\end{array}$ & $\begin{array}{l}* * * \\
* * * \\
* * * \\
* *\end{array}$ \\
\hline $\begin{array}{l}\text { Régions d'Italie } \\
\text { Nord } \\
\text { Centre } \\
\text { Sud }\end{array}$ & $\begin{array}{l}-.14 \\
-.24 \\
-.38\end{array}$ & $\begin{array}{l}\text { n.s. } \\
* * * \\
* * *\end{array}$ & $\begin{array}{l}-.62 \\
1.24 \\
1.27\end{array}$ & $\begin{array}{l}\mathrm{n} . \mathrm{s} . \\
* * * \\
* * *\end{array}$ & $\begin{array}{l}-.06 \\
-.35 \\
1.87\end{array}$ & $\begin{array}{l}\mathrm{n} . \mathrm{s} . \\
* * * \\
* * *\end{array}$ & $\begin{array}{l}-.86 \\
1.66 \\
1.84\end{array}$ & $\begin{array}{l}* \\
* * * \\
* * *\end{array}$ \\
\hline $\begin{array}{l}\text { Source: Panel eur } \\
\text { Champ: populatio } \\
(*): \mathrm{P}<.1, *: \mathrm{P}<. \\
\text { Note }: \text { Le modèle } \\
\text { cation et du reven }\end{array}$ & $\begin{array}{l}\text { ménas } \\
65 \text { ans } \\
<.0 .1, \\
\text { ffet de } \\
\text { ge }\end{array}$ & $\begin{array}{l}1994 \\
\mathrm{P}<. \\
\text { ge, d }\end{array}$ & $\begin{array}{l}\text { gue } \\
\text {; n.s } \\
\text { xe, d }\end{array}$ & $\begin{array}{l}\text { on sign } \\
\text { compo }\end{array}$ & $\begin{array}{l}\text { icatif } \\
\text { ition du }\end{array}$ & enc & niv & l'édu- \\
\hline
\end{tabular}

uniquement les aides financières reçues de personnes vivant en dehors du ménage. On peut considérer que le besoin d'obtenir de telles aides est plus fort pour les chômeurs de longue durée puisqu'ils sont, parmi les actifs, les plus démunis, étant donné que le montant de l'indemnisation du chômage diminue en fonction de la durée du chômage.

Par rapport à la modalité de référence dans le modèle, à savoir les personnes en emploi stable, le coefficient pour les chômeurs de longue durée est positif, élevé et très significatif dans les pays du Sud de l'Europe, Italie, Espagne, Portugal et Grèce, alors qu'il est moins élevé en Belgique ou non significatif en France, au Danemark, en Grande-Bretagne et en Irlande. On peut donc conclure que les chômeurs de longue durée du Sud de l'Europe sont plus aidés par leur entourage, y compris après avoir contrôlé l'effet de plusieurs variables: le sexe, l'âge, la composition du ménage, le niveau d'éducation et le revenu du ménage.

Le cas des Pays-Bas mérite une attention particulière: le coefficient pour les chômeurs de longue durée est approximativement le même que dans les pays du Sud de l'Europe, ce qui est surprenant puisque les transferts sociaux assurés par l'Etat-providence de ce pays y sont nettement plus élevés. Il aurait été, en effet, 
logique d'observer, en raison de l'efficacité globale du système néerlandais de protection sociale, un moindre besoin des chômeurs d'avoir recours aux aides privées ou, de façon plus précise, un besoin moins fondamental pour survivre. $\mathrm{Ce}$ résultat peut s'expliquer par le fait, vérifié par les sociologues néerlandais, que les chômeurs restent vivre à proximité de leur famille et sont moins mobiles géographiquement que les autres actifs. On notera toutefois que, dans l'ensemble, la proportion de chômeurs de longue durée aidés par leur famille ou leur entourage reste globalement faible (moins de 1\%). C'est donc uniquement en termes relatifs que les solidarités familiales des chômeurs de longue durée des Pays-Bas se rapprochent de celles dont bénéficient les chômeurs de longue durée des pays du Sud de l'Europe. Notons aussi que les chômeurs de moins d'un an sont également, de façon significative, davantage aidés par leur entourage que les personnes en emploi stable dans les pays du Sud, ce qui n'est pas le cas aux Pays-Bas.

En Italie, les différences entre régions sont profondes. Dans le Nord, l'effet de la précarité de l'emploi (emploi menacé, emploi instable) et du chômage de moins d'un an sur l'aide de la famille et de l'entourage n'est pas significatif par rapport à l'emploi stable. En revanche, il y a un effet fort et significatif dans le Centre et le Sud pour chacune des quatre situations par rapport à l'emploi, de l'emploi menacé au chômage de longue durée. Les coefficients sont toujours plus élevés dans le Sud de l'Italie. Ces résultats peuvent être expliqués par le faible développement économique de cette région et par l'organisation locale des familles pauvres.

Bien que la question posée concernait l'aide reçue de l'extérieur du ménage, il convient de souligner qu'elle peut compléter la solidarité qui s'exerce aussi au sein du ménage puisque, on l'a vu, les chômeurs sont plus nombreux à vivre chez leurs parents. Ces solidarités se maintiennent-elles lorsque les chômeurs vivent seuls? Pour y répondre, on a donc calculé la proportion de chômeurs vivant seuls aidés par leur famille ou leur entourage et la même proportion pour les personnes vivant seuls, mais non au chômage. Le résultat est que les chômeurs vivant seuls sont, une fois encore, proportionnellement plus nombreux à être aidés en Grèce, en Espagne et en Italie. En Grèce, plus d'un chômeur vivant seul sur deux reçoit une aide de ce type.

Si dans les pays du Sud de l'Europe, les solidarités familiales jouent un rôle important dans l'atténuation du risque de pauvreté qui découle du chômage, cela ne veut pas dire que le rôle des aides versées au titre de la solidarité nationale dans les pays du Nord a pour effet de dissoudre le soutien apporté par la famille. Il faut y voir avant tout l'effet d'un système social. Quand une grande partie de la population partage la même condition sociale défavorisée, la solidarité familiale s'inscrit avant tout dans une logique de lutte collective contre la pauvreté. La réciprocité dans l'échange est alors fonctionnelle. Chacun donne et rend, car chacun se donne et se rend pour faire face à l'épreuve. C'est la raison pour laquelle dans les régions où le chômage et la pauvreté sont très élevés, il y a plus de chances de trouver des solidarités familiales de longue durée fondées sur la réciprocité imposée par la nécessité de résister collectivement ${ }^{15}$.

${ }^{15}$ Ce constat s'appuie notamment sur la théorie de Mauss sur le don qui reste encore particulièrement féconde pour analyser les solidarités familiales. On pourra se référer sur ce point à l'article suivant: S. Paugam et J.-P. Zoyem, «Le soutien financier de la famille: une forme essentielle de la solidarité », Economie et Statistique, n 308-309-310, 1997, 8/9/10, pp. 187-120. 
Si les solidarités familiales n'ont pas disparu dans les régions plus développées économiquement, elles n'y ont pas cette fonction vitale. La volonté d'autonomie des individus et le caractère moins homogène des familles rendent, dans l'ensemble, les solidarités familiales à la fois plus souples, plus informelles, mais aussi plus fragiles. Lorsque les échanges au sein de la famille deviennent profondément inégalitaires, ils risquent d'empêcher les bénéficiaires de donner et de se donner à leur tour, ce qui, à terme, ne peut que les disqualifier.

\section{CHÔMAGE ET DÉCLIN DU LIEN DE PARTICIPATION ÉLECTIVE}

Pour appréhender l'isolement social, on peut prendre en compte aussi la participation à la vie associative qui correspond, d'après notre classification, à un lien de participation élective. Depuis l'enquête de Lazarsfeld à Marienthal au début des années 1930, on sait que le chômage contribue à affaiblir l'intensité des échanges sociaux, en particulier dans la sphère des relations associatives. Les clubs de théâtre, les clubs sportifs, les associations bénévoles connurent, en effet, un déclin significatif à partir du moment où la grande partie de la population de cette ville moyenne fut touchée par la fermeture de la principale usine. En réalité Les chômeurs de Marienthal est un livre qui se lit comme le récit d'une catastrophe sociale. Les auteurs nous invitent en effet à pénétrer dans ce village, à y découvrir cette morne indifférence qui se dégage de ces lieux presque abandonnés: «ici vivent des gens qui se sont habitués à posséder moins, à entreprendre moins et à espérer moins que ce qui est considéré comme nécessaire à une existence ordinaire.» ${ }^{16}$ Le travail à l'usine était le centre de la vie sociale au sens où il apportait aux travailleurs non seulement une activité et une rémunération, mais une raison d'être, un sentiment d'utilité et une reconnaissance sociale. D'une façon générale, c'est la communauté dans son ensemble qui est devenue lasse. Le rétrécissement du champ d'activité a transformé le fonctionnement de certaines institutions (la bibliothèque municipale, les clubs de loisirs, le théâtre etc.) et a atteint progressivement la vie privée des chômeurs.

Dans L'épreuve du chômage Dominique Schnapper a également insisté sur l'affaiblissement de la vie sociale des chômeurs. L'effet du chômage sur la vie sociale semble en effet très comparable pour certaines personnes comme on peut le constater dans ces deux extraits d'entretiens recueillis pourtant à près de quarante ans d'intervalle:

\footnotetext{
«Je passe la plupart de mon temps chez moi. Depuis que je suis au chômage, je ne lis presque plus. On n'a pas la tête à ça » (Les chômeurs de Marienthal, cf. p. 71)

«On est plus seul. Oui, dans le temps, j’avais des camarades, on allait à la pêche, on allait... eh bien maintenant, je préfère être seul que de... [...] on s'aigrit, on s'aigrit [...]. Dans le temps, j'aimais encore blaguer. Eh bien maintenant, c'est fini. C'est un machin... on n'est pas toujours réellement dans nos assiettes, ça travaille.» (L'épreuve du chômage, p. 144).
}

${ }^{16}$ Cf. Les chômeurs de Marienthal, op. cit., p. 67. 
Qu'en est-il aujourd'hui? Les exploitations du European Community Household Panel ont permis de vérifier que le chômage avait toujours un effet négatif sur la vie associative à la fin du $\mathrm{XX}^{\mathrm{e}}$ siècle et cela dans les principaux pays européens ${ }^{17}$. On peut constater, par exemple, dans le tableau 3 que la participation des chômeurs à la vie associative est plus faible que celle des personnes ayant un emploi stable, aussi bien dans les pays du $1^{\text {er }}$ groupe que dans les trois autres. Notons cependant que la proportion de chômeurs de plus d'un an qui participent à la vie associative est relativement élevée au Danemark comparativement aux autres pays: ils sont en effet $47,6 \%$ dans ce pays contre seulement $33 \%$ au Royaume-Uni, 29,5\% en Allemagne, 18,8\% en France et 9,3\% au Portugal. On peut expliquer ces différences par les formes de sociabilité qui restent contrastées d'un pays à l'autre. Elles sont plus informelles et plus organisées à l'échelon de la famille et du réseau de proximité dans les pays du Sud. Mais en dépit de cette différence, on observe partout une tendance à une moindre vie sociale des chômeurs. On ne peut toutefois s'en tenir à cette simple comparaison des proportions sans neutraliser les éventuels effets de structure.

Tableau 3 : Participation à la vie associative selon la situation par rapport à l'emploi (en \%).

\begin{tabular}{|c|c|c|c|c|}
\hline Pays & Emploi stable & $\begin{array}{l}\text { Chômage de } \\
\text { moins d'un an }\end{array}$ & $\begin{array}{l}\text { Chômage de } \\
\text { plus d'un an }\end{array}$ & $\begin{array}{l}\text { Ensemble de la } \\
\text { population active } \\
\text { de } 18 \text { à } 65 \text { ans }\end{array}$ \\
\hline $\begin{array}{l}\mathbf{1}^{\mathrm{er}} \text { groupe } \\
\text { Danemark } \\
\text { Pays-Bas }\end{array}$ & $\begin{array}{l}61,1 \\
51,2\end{array}$ & $\begin{array}{l}43,0 \\
46,1\end{array}$ & $\begin{array}{l}47,6 \\
39,0\end{array}$ & $\begin{array}{l}58,8 \\
50,0\end{array}$ \\
\hline $\begin{array}{l}2^{\mathrm{e}} \text { groupe } \\
\text { Allemagne } \\
\text { France } \\
\text { Belgique }\end{array}$ & $\begin{array}{l}57,3 \\
31,4 \\
38,1\end{array}$ & $\begin{array}{l}27,3 \\
22,5 \\
32,6\end{array}$ & $\begin{array}{l}29,5 \\
18,8 \\
18,7\end{array}$ & $\begin{array}{l}51,8 \\
28,0 \\
35,5\end{array}$ \\
\hline $\begin{array}{l}\mathbf{3}^{\mathrm{e}} \text { groupe } \\
\text { Roy. Uni } \\
\text { Irlande }\end{array}$ & $\begin{array}{l}49,3 \\
53,2\end{array}$ & $\begin{array}{l}44,2 \\
37,1\end{array}$ & $\begin{array}{l}33,0 \\
21,0\end{array}$ & $\begin{array}{l}48,5 \\
47,5\end{array}$ \\
\hline $\begin{array}{l}\mathbf{4}^{\mathrm{e}} \text { groupe } \\
\text { Italie } \\
\text { Espagne } \\
\text { Grèce } \\
\text { Portugal }\end{array}$ & $\begin{array}{l}21,5 \\
34,5 \\
14,9 \\
20,1\end{array}$ & $\begin{array}{l}12,7 \\
22,8 \\
10,5 \\
11,6\end{array}$ & $\begin{array}{l}11,8 \\
21,6 \\
10,4 \\
9,3\end{array}$ & $\begin{array}{l}18,4 \\
30,4 \\
12,9 \\
18,3\end{array}$ \\
\hline
\end{tabular}

Le tableau 4 permet d'analyser l'effet propre du chômage de moins d'un an et de plus d'un an sur la participation à la vie associative par rapport à la modalité de référence, les personnes ayant un emploi stable. Comme dans le tableau 2 ci-dessus, les effets de l'âge du sexe, de la composition du ménage du niveau d'éducation et du

${ }^{17}$ Pour une étude plus approfondie de cette question, on pourra se reporter à l'étude de S. Paugam et de H. Russell, «The Effects of Unemployment Precarity and Unemployment on Social Isolation », in D. Gallie and S. Paugam (dir.) Welfare Regimes and the Experience of Unemployment in Europe, op. cit., pp. 243-264. 
Tableau 4 : Effets de l'instabilité de l'emploi et du chômage sur la participation à la vie associative (régression logistique) Référence : emploi stable

\begin{tabular}{|c|c|c|c|c|c|c|c|c|}
\hline \multirow[b]{2}{*}{ Pays } & \multicolumn{2}{|c|}{ Emploi menacé } & \multicolumn{2}{|c|}{ Emploi instable } & \multicolumn{2}{|c|}{ Chômage $<1$} & \multicolumn{2}{|c|}{ Chômage $>1$} \\
\hline & B. & Sig. & B. & Sig. & B. & Sig. & B. & Sig \\
\hline $\begin{array}{l}\mathbf{1}^{\mathrm{er}} \text { groupe } \\
\text { Danemark } \\
\text { Pays-Bas }\end{array}$ & $\begin{array}{l}-.12 \\
-.04\end{array}$ & $\begin{array}{l}* * \\
\mathrm{~ns}\end{array}$ & $\begin{array}{l}-.10 \\
-.40\end{array}$ & $\begin{array}{l}\text { Ns } \\
* *\end{array}$ & $\begin{array}{l}-.62 \\
-.20\end{array}$ & $\begin{array}{l}* * * \\
\mathrm{~ns}\end{array}$ & $\begin{array}{l}-.30 \\
-.43\end{array}$ & $\begin{array}{l}\mathrm{ns} \\
* *\end{array}$ \\
\hline $\begin{array}{l}2^{\mathrm{e}} \text { groupe } \\
\text { Allemagne } \\
\text { France } \\
\text { Belgique }\end{array}$ & $\begin{array}{l}-.42 \\
-.03 \\
-.24\end{array}$ & $\begin{array}{l}* * * \\
\mathrm{~ns} \\
* *\end{array}$ & $\begin{array}{l}-.73 \\
-.09 \\
-.33\end{array}$ & $\begin{array}{l}* * * \\
\mathrm{~ns} \\
\mathrm{~ns}\end{array}$ & $\begin{array}{l}1.05 \\
-.21 \\
-.14\end{array}$ & $\begin{array}{l}* * * \\
* \\
\mathrm{~ns}\end{array}$ & $\begin{array}{l}-.96 \\
-.37 \\
-.79\end{array}$ & $\begin{array}{l}* * * \\
* \\
* * *\end{array}$ \\
\hline $\begin{array}{l}\mathbf{3}^{\mathrm{e}} \text { groupe } \\
\text { Roy.Uni } \\
\text { Irlande }\end{array}$ & $\begin{array}{l}-.09 \\
-.24\end{array}$ & $\begin{array}{l}\mathrm{ns} \\
* *\end{array}$ & $\begin{array}{l}-.27 \\
-.17\end{array}$ & $\begin{array}{l}(*) \\
\mathrm{ns}\end{array}$ & $\begin{array}{l}-.12 \\
-.05\end{array}$ & $\begin{array}{l}\text { ns } \\
\text { ns }\end{array}$ & $\begin{array}{l}-.51 \\
-.57\end{array}$ & $\begin{array}{l}* * * \\
* * *\end{array}$ \\
\hline $\begin{array}{l}4^{\text {e }} \text { groupe } \\
\text { Italie } \\
\text { Espagne } \\
\text { Grèce } \\
\text { Portugal }\end{array}$ & $\begin{array}{l}-.24 \\
-.08 \\
-.08 \\
-.13\end{array}$ & $\begin{array}{l}* * * \\
\mathrm{~ns} \\
\mathrm{~ns} \\
\mathrm{~ns}\end{array}$ & $\begin{array}{l}-.47 \\
-.06 \\
-.03 \\
-.29\end{array}$ & $\begin{array}{l}* * \\
\mathrm{~ns} \\
\mathrm{~ns} \\
\mathrm{~ns}\end{array}$ & $\begin{array}{l}-.50 \\
-.19 \\
-.08 \\
-.09\end{array}$ & $\begin{array}{l}* * * \\
* * \\
\mathrm{~ns} \\
\mathrm{~ns}\end{array}$ & $\begin{array}{l}-.50 \\
-.26 \\
-.01 \\
-.10\end{array}$ & $\begin{array}{l}* * * \\
* * \\
\mathrm{~ns} \\
\mathrm{~ns}\end{array}$ \\
\hline $\begin{array}{l}\text { Source: Par } \\
\text { Champ: po } \\
(*): \mathrm{P}<.1, \\
\text { Note } \text { Le } \mathrm{m} \\
\text { cation et du }\end{array}$ & $\begin{array}{l}\text { ménas } \\
65 \text { ans } \\
<.0 .1, \\
\text { effet d } \\
\text { ge }\end{array}$ & $\begin{array}{l}199 \\
\mathrm{P}< \\
\text { age, }\end{array}$ & $\begin{array}{l}\text { gue } \\
\text {, ns: } \\
\text { exe, }\end{array}$ & a comp & $\begin{array}{l}\text { atif } \\
\text { sition }\end{array}$ & lén & du ni & d'édu- \\
\hline
\end{tabular}

revenu du ménage sont contrôlés. Il est frappant tout d'abord de constater que la valeur des coefficients, aussi bien pour le chômage de moins d'un an que pour le chômage de plus d'un an, est presque systématiquement négative. Après contrôle des variables sociodémographiques de base, on peut donc conclure que, globalement, les chômeurs participent moins à la vie associative que les personnes ayant un emploi stable. Mais la valeur elle-même des coefficients et la significativité statistique changent d'un pays à l'autre. Dans le cas du Danemark, c'est seulement pour les chômeurs de moins d'un an que le coefficient est significatif (en grande partie parce qu'il existe peu de chômeurs de longue durée dans ce pays). En revanche, en Belgique, au Royaume-Uni et en Irlande, les coefficients ne sont significatifs que pour les chômeurs de plus d'un an. Dans ces pays, c'est seulement dans la durée que l'effet de l'affaiblissement de la vie sociale est visible.

Enfin, soulignons que dans les deux pays les moins économiquement développés, où la proportion de pauvres est la plus élevée, à savoir la Grèce et le Portugal, les coefficients sont très faibles et non significatifs aussi bien pour le chômage de moins d'un an que pour le chômage de plus d'un an. Ils ne le sont pas non plus pour l'emploi menacé ou instable. La thèse du chômage total n'est donc pas vérifiée systématiquement.

Pour interpréter ces différences, il faut tenir compte du statut du chômage dans chaque société. Lorsque le chômage est massif depuis plusieurs années et lié, 
comme c'est le cas dans plusieurs pays ou régions du Sud de l'Europe, à la pauvreté et au faible développement économique, il correspond à une condition banale à laquelle tout le monde est ou peut être confronté. Dans ce cas, il a peu de chances d'affecter profondément les relations sociales, lesquelles peuvent avoir pour fondement justement de conjurer la misère, c'est-à-dire d'offrir à chaque individu des moyens de résistance psychologique et des modes de participation informels aux échanges. Dans les pays qui ont connu le plein emploi et dont la situation de l'emploi est aujourd'hui dégradée, les chômeurs ont plus de chances de connaître l'épreuve de la disqualification sociale, d'autant que les représentations de l'honneur social sont plus souvent fondées sur le statut que procure la participation directe à l'activité professionnelle

\section{TROIS MODÈLES DE RÉGULATION DU CHÔMAGE}

Ces différences entre pays montrent qu'il n'y pas une relation simple entre chômage, pauvreté et rupture cumulative des liens sociaux. Au contraire, nous suggérons que la probabilité de cumul de handicaps dépend du modèle de régulation sociale du chômage qui prévaut dans la société. Les modèles de régulation sociale du chômage peuvent être appréhendés à partir de la relation entre, d'une part, les responsabilités attribuées à la sphère publique d'intervention de l'Etatprovidence (qui renvoie notamment aux garanties offertes par le lien de citoyenneté) et, d'autre part, les responsabilités qui relèvent de la sphère d'intervention de la famille (qui renvoie en grande partie au lien de filiation). Ces modèles sont des types idéaux, élaborés pour analyser de façon compréhensive des différences dans les principes fondamentaux d'organisation sociale. Bien que des sociétés particulières peuvent être proches de l'un ou de l'autre de ces modèles, nous ne pouvons attendre qu'une société reflète entièrement l'un d'entre eux dans sa pure forme. Nous pouvons distinguer trois types de modèles principaux: le modèle public individualiste, le modèle de responsabilité partagée et le modèle familialiste.

Le modèle public individualiste repose sur l'hypothèse que la société dans son ensemble a la responsabilité du problème du chômage et, par conséquent, du bienêtre des chômeurs. Puisque ces derniers n'ont pas à assumer la responsabilité de leur situation personnelle, l'objectif du système d'Etat-providence est prioritairement de garantir leur niveau de vie. Cela implique un haut niveau de développement du système de protection sociale, lequel fournira également des ressources pour assurer une participation a la vie sociale indépendamment de la situation des individus sur le marché de l'emploi et vis-à-vis de la famille. Etant donné le haut niveau de l'aide publique, l'obligation normative des familles de prendre en charge leurs membres lorsqu'ils sont au chômage est faible. Puisque la responsabilité du chômage est attribuée à la société plutôt qu'à l'individu, c'est un modèle dans lequel le chômage a peu de chances de déterminer les relations sociales entre l'individu et la communauté locale.

Le modèle familialiste, au contraire, rejette le principe de base d'une responsabilité sociale collective face au chômage, mais maintient une forte exigence à l'égard des devoirs de la famille en ce qui concerne la prise en charge de ses membres, au double sens du ménage et du réseau de parenté élargie. Le rôle de la politique publique est avant tout de préserver l'intégrité de la famille contre tous 
les risques de remise en question de sa fonction protectrice. L'hypothèse implicite est encore que l'individu n'est pas à blâmer en raison de sa situation de chômage et qu'il a donc un droit à partager les ressources de sa famille pendant la période où il est en difficulté. Les responsabilités à l'égard des chômeurs sont semblables dans ce cas aux responsabilités à l'égard des enfants dépendants. En raison des implications quotidiennes de la vie familiale et de ses formes ordinaires de sociabilité, le chômage a également peu de chances de se traduire par une réduction des relations sociales dans la communauté.

Enfin, le modèle de responsabilité partagée se caractérise par la recherche d'un équilibre entre la prise en charge des chômeurs qui revient aux autorités publiques et celle qui revient à la famille. Il est possible de définir les frontières de ces responsabilités de plusieurs façons. Elles peuvent être synchroniques et se traduire notamment par une intervention publique pour assurer les besoins de base et une intervention de la famille pour assurer une protection plus large du niveau de vie. De façon alternative, dans le cas du chômage, la relation peut être définie temporairement de façon à permettre successivement une prise en charge des chômeurs par les ressources publiques, en particulier dans la première phase du chômage, et ensuite par les ressources de la famille dans les phases suivantes. L'hypothèse implicite de tels systèmes est que l'individu peut être, au mois partiellement, responsable de sa situation. Les limites de l'intervention des pouvoirs publics traduisent en elles-mêmes une sorte de suspicion à l'égard des personnes qui pourraient avoir tendance à préférer le chômage à l'emploi. De ce fait, on attire l'attention sur les effets éventuellement désincitatifs que pourraient avoir une protection substantielle de leur niveau de vie dans le temps. Le rôle résiduel attribué à la famille implique par ailleurs que ce n'est pas non plus une responsabilité que la famille aurait normalement à assumer. Dans ces conditions, le soutien de la famille a des chances d'être accompagné de fortes pressions sur l'individu pour qu'il accède - ou re-accède - au marché de l'emploi. Le soutien de la famille prend alors la forme d'un système de contrôle social des chômeurs. Compte tenu des conditions restrictives de l'aide publique et de l'importance accordée à l'idée de responsabilité potentielle de l'individu, il existe une forte probabilité que la pauvreté et le chômage affectent profondément l'identité de soi et se traduisent progressivement par un retrait de la vie sociale.

Ces différents modes de régulation sociale du chômage peuvent avoir des conséquences en termes de ruptures des liens sociaux. Le modèle public individualiste aura tendance à atténuer les effets du chômage en fournissant une protection du niveau de vie relativement haute. Il peut donc permettre aux personnes de continuer à participer à la vie associative (lien de participation élective) et de participer en tant que citoyens aux affaires de la Cité (lien de citoyenneté). Toutefois, la dimension individualiste qui caractérise ce modèle peut aussi accroître le risque d'isolement social dans la sphère des relations plus intimes (lien de filiation). On peut expliquer ce processus en partie par les conditions matérielles qui autorisent ces personnes à se maintenir dans des ménages indépendants, mais en partie aussi par la faible pression normative exercée sur le réseau de parenté pour apporter une aide financière ou affective.

Le modèle familialiste aura tendance à apporter une aide relativement minime pour assurer le niveau de vie. Les ressources familiales existantes seront dans ce cas seulement mieux réparties pour faire face aux besoins de l'ensemble des 
membres du réseau de parenté. Lorsque les chômeurs sont issus des milieux peu qualifiés et relativement défavorisés, comme c'est le cas fréquemment, la responsabilité familiale de leur prise en charge peut entraîner un grand risque d'appauvrissement de la famille tout entière. Toutefois, le modèle familialiste protège d'une certaine manière contre la disqualification sociale en garantissant aux chômeurs une forme de sociabilité. C'est en partie la conséquence du fait que les chômeurs ont une forte probabilité de vivre avec leurs parents, si bien qu'ils ont ainsi des contacts réguliers et des relations sociales quotidiennes dans leur cadre de vie ordinaire correspondant à leur lieu de résidence. Ce processus s'explique également par la forte pression normative exercée sur le réseau de parenté pour prendre en charge les besoins des membres défavorisés de la famille.

Le modèle de responsabilité partagée est celui qui se traduit par le risque le plus élevé de rupture cumulative des liens sociaux. D'une certaine manière, on peut dire que ce risque est lié directement à la définition sociale implicite du chômage qui met l'accent sur les défaillances potentielles des chômeurs. Dans la sphère d'intervention de l'Etat providence, la suspicion à l'égard d'une frange d'entre eux conduit à ne pas les traiter comme des citoyens égaux. Dans la sphère d'intervention de la famille, les chômeurs ne sont pas non plus toujours considérés comme des membres du réseau de parenté aussi méritants que les autres. Ce modèle fondé sur la méfiance conduit inéluctablement à des réactions défensives et conflictuelles dans les relations interpersonnelles. Toutefois, c'est aussi parce que ce système n'est pas fondé sur une structure normative clairement définie qu'il rend en permanence possibles des conflits d'ordre normatif. Ces derniers peuvent apparaître notamment lorsqu'il s'agit de définir un critère rationnel pour déterminer à quel moment l'aide publique doit cesser et l'aide de la famille commencer. Il en ressort presque inévitablement un désaccord chronique sur les responsabilités légitimes dans ce domaine. La famille peut être encouragée à se désengager de ce type de prise en charge, souvent considérée comme un fardeau, en particulier lorsqu'elle estime que celui-ci pourrait être supporté par l'Etat. Et si elle continue malgré tout à apporter son soutien, il est possible que ce soit à la longue à contre coeur, ce qui ne peut qu'accroître les tensions familiales. Il existe enfin un risque beaucoup plus élevé que le bien-être des chômeurs en soit fortement affecté puisque, dans ses interstices, ce système peut laisser des personnes à la fois sans aucune ressource en provenance de l'Etat providence et sans aide et soutien affectif de la famille.

L'ampleur des problèmes associés au modèle de responsabilité partagée dépend clairement à la fois de la nature spécifique du système d'Etat providence et de l'éthos de la famille. Lorsque ce modèle est associé à un niveau de compensation relativement haut sur une longue durée, il affectera moins le bien-être individuel que s'il prend la forme d'un très faible niveau ou d'une courte durée de l'aide apportée par l'Etat providence. Par ailleurs, lorsque, pour des raisons d'ordre religieux, les normes familiales sont particulièrement fortes, la famille aura plus de chances d'offrir une aide pour compenser les lacunes de l'intervention de l'Etat que dans les sociétés ou les conceptions traditionnelles de la famille ont plus ou moins disparu. Pour ces raisons à la fois institutionnelles et culturelles, on peut attendre de fortes variations entre les pays qui peuvent être globalement considérés comme les plus proches du modèle de responsabilité partagée.

En Europe, les pays scandinaves sont les seuls à remplir les conditions d'un haut niveau d'autonomie individuelle. Cela peut avoir un coût en termes d'isolement 
social, en particulier dans la sphère du ménage. Mais, d'une façon générale, les résultats confirment qu'un tel système présente un faible risque de rupture cumulative des liens sociaux. A l'autre extrémité, ce sont les pays du Sud de l'Europe qui se rapprochent le plus du modèle familialiste de régulation sociale. Les chômeurs sont confrontés à de grandes difficultés financières et ne disposent que d'un très faible revenu individuel, mais socialement ils restent fortement intégrés à la fois en termes de relations dans le ménage - puisqu'ils sont nombreux à vivre avec leurs parents et à participer à la vie sociale de la famille - et en termes de sociabilité avec leurs voisins et amis avec qui ils forment le plus souvent une vraie communauté. Pour résumer, le lien de filiation et le lien de participation élective se maintiennent.

Les problèmes du modèle de responsabilité partagée apparaissent le plus clairement dans les pays qui ont adopté un régime d'indemnisation intermédiaire entre les pays du Nord et les pays du Sud. Mais l'importance de ces effets peut varier selon la générosité du système de protection sociale et la force des valeurs familiales. En Irlande, non seulement les transferts sociaux sont relativement plus importants qu'au Royaume-Uni, mais le risque d'isolement social est fortement réduit en raison de la force du lien de filiation - sans doute liée à l'influence du catholicisme - et de son effet sur le rôle protecteur de la famille. Cette tendance se traduit par un niveau d'intégration sociale proche de celui des pays du Sud. Les Pays-Bas et la Belgique sont plus généreux pour les chômeurs en termes de compensation financière, ce qui se traduit par des niveaux intermédiaires de cumul des deux handicaps de pauvreté et d'isolement social. Il est clair que le risque de cumul de ces deux handicaps est le plus élevé dans les pays où les responsabilités normatives respectives des autorités publiques et de la famille sont les moins clairement définies. L'Allemagne, la France et le Royaume-Uni sont les pays où le niveau de l'aide financière publique est intermédiaire et, dans le cas du RoyaumeUni, très bas. Ce sont aussi, en même temps, les pays dans lesquels les responsabilités institutionnelles de la famille sont relativement peu définies. C'est, par conséquent, dans ces pays que les chômeurs risquent le plus d'être confrontés simultanément à la pauvreté et à la rupture des liens sociaux, ce que nous pouvons considérer comme des conditions favorisant le processus de disqualification sociale. 